\title{
Insulin Antagonizes Thrombin-Induced Microvessel Leakage
}

\author{
Yan Liu $^{\mathrm{a}} \quad$ Xue lian Chen ${ }^{\mathrm{b}}$ Lei Wang ${ }^{\mathrm{a}}$ Manuela Martins-Green ${ }^{\mathrm{a}}$ \\ ${ }^{\text {a }}$ Department of Cell Biology and Neuroscience, University of California Riverside, Riverside, CA, USA; ${ }^{b}$ Department of \\ Burn and Plastic Surgery, Shanghai JiaoTong University School of Medicine, Ruijin Hospital, Shanghai, PR China
}

\section{Keywords}

Signal transduction - Vascular biology · Shock resuscitation · Inflammatory response $\cdot$ Sepsis

\begin{abstract}
Sustained increase in microvessel permeability results in cell and tissue damage. To date, it has not been possible to safely and specifically block increased microvessel permeability in vivo. We showed that insulin stimulates angiogenesis and that the new microvessels are associated with more aSMAproducing cells, suggesting greater stability. In this study, we show that local injection of insulin under the skin of mice significantly inhibits thrombin-induced microvessel permeability and that insulin improves the barrier function of primary human endothelial cells under conditions that mimic endothelium in vivo. These findings indicate that insulin antagonizes thrombin-induced microvessel permeability. At the cell and molecular levels, we show that insulin interferes with thrombin-induced VE-cadherin signaling by decreasing the ability of thrombin to induce VE-cadherin translocation to the cytoskeleton/nuclear compartment, leading to microvessel leakage. Simultaneously, the rapid activation of Src by insulin followed by the activation of Rac1, a small GTPase involved in cytoskeletal reorganization, leads to the maintenance of endothelial barrier, short-circuiting the slower thrombin-induced Src-RhoA signaling that leads to endo-
\end{abstract}

\section{KARGER}

(C) 2017 S. Karger AG, Basel

E-Mail karger@karger.com

www.karger.com/jvr thelial permeability. This novel mechanism by which insulin inhibits thrombin-induced permeability provides support for the use of insulin treatment in pathological conditions that involve blood-barrier dysfunction, especially as resuscitation treatment methods for extensive burns, sepsis, and other severe pathological conditions. @ 2017 S. Karger AG, Basel

\section{Introduction}

A large number of inflammatory mediators and vasoactive agents are generated when tissue injury occurs and when inflammation ensues. They stimulate an increase in blood vessel permeability during traumatic situations, infections, and metabolic dysfunction in many acute and chronic diseases, such as sepsis [1], severe trauma [2] including extensive burns [3], and diabetes [4]. Controlled increase in vessel permeability has beneficial effects, including increase in the supply of oxygen and nutrients to the tissues and increase in leukocyte trafficking during inflammatory responses. Increased permeability also increases the deposition of fibrin that provides a scaffold for inflammatory and repair cells to migrate and proliferate. However, uncontrolled and lasting increases in permeability frequently cause loss of electrolytes and proteins and insufficient volume in the circulatory system, as well

Dr. Manuela Martins-Green

Department of Cell Biology and Neuroscience

University of California Riverside

Riverside, CA 92521 (USA)

E-Mail manuela.martins@ucr.edu 
as tissue edema, elevated interstitial pressure, ischemia, and anaerobic metabolism, resulting in extensive cell and tissue damage.

Early stages of severe burns are typically characterized by increased vessel permeability causing loss of circulating body fluid potentially leading to shock. It has been reported in a retrospective study with more than 12,000 cases that the incidence of burn-induced shock was $85 \%$ when the burn area exceeds $90 \%$ TBSA; in the case of 50-69\% TBSA, the ratio reached 57\% [5]. Delayed resuscitation is closely correlated with systemic infection, burn sepsis, and multiple organ dysfunction syndrome [6]. Another common pathological situation in which vessel permeability changes are observed is infection-induced sepsis, a systemic inflammatory response caused by infection that may originate from many different diseases and pathological condition. In this case, elevated levels of inflammatory mediators induce extensive and increased capillary permeability leading to lethal septic shock. From a large retrospective analysis that included 500 nonfederal US hospitals and more than 10 million cases of sepsis, the National Center for Health Statistics reported a threefold increase of sepsis incidence over a 22 -year period with the highest rate of 2.4 cases/1,000 people/year [7]. In patients with septic shock, mortality rates ranged from 40 to $75 \%$ based on different reports [8]. Unfortunately, to date, no specific treatment targeted to decrease blood vessel permeability is available clinically, although the demand is urgent considering the high incidence of shock and subsequent complications.

Thrombin is one of the very effective permeability increasing factors in situations of shock or sepsis [1]. It is also known that in critically ill burn patients and those with severe large burns, there is significant disseminated intravascular coagulation indicating that pro-thrombin is activated to thrombin shortly after severe burns and contributes to the increase in vessel permeability observed under these conditions $[9,10]$. Therefore, development/ identification of a molecule(s) that antagonizes the severe effects of thrombin on increased vessel permeability would represent a major advancement in alleviating these serious conditions. Thrombin binds to heterotrimeric GPCRs and activates them by proteolytic cleavage of the $\mathrm{N}$-terminus, hence the name proteolytically activated receptors (PARs) [11]. Thrombin activation of PAR-1 in endothelial cells results in multiple downstream signals that stimulate Rho kinase causing actin stress fiber formation, which leads to endothelial cell-shape changes and gap formation. Another molecular target for thrombin is VE-cadherin, a membrane protein predominantly ex- pressed in endothelial cells, which is crucial in stabilizing the endothelium, as well as in regulating the barrier between the circulating blood cells and the surrounding tissues [12].

For effective treatment, early resuscitation, as suggested by the "Surviving Sepsis Campaign Guidelines Committee," must be applied [13]. It is known that this approach has saved thousands of lives by perfusion of several thousand milliliters of solution, including plasma, albumin, and crystalloid solution within several hours after sepsis shock occurs. However, all treatments for resuscitation are still symptomatically targeted and not etiologically targeted because to date not a single drug has been found that can be safely applied to specifically block increased vessel permeability in vivo. Therefore, finding an effective treatment to control and/or antagonize increased vessel permeability would make a very significant contribution to the treatment of many diseases in which increased vessel permeability is a critical component. Our previous work showed that insulin stimulates angiogenesis through the PI3K-Akt-Rac1 signal pathway and that the microvessels have many more aSMA-producing cells associated with them than controls, suggesting that it stimulates vessel stability potentially increasing barrier function [14]. These results have been subsequently confirmed by others $[15,16]$. Therefore, we hypothesize that the ability of insulin to increase barrier function (i.e., decrease vessel permeability) results in antagonizing the function of molecules such as thrombin, which stimulate the breakdown of barrier function (i.e., increase vessel permeability). We show that insulin interferes with thrombin-induced VE-cadherin translocation to the cytoskeleton/nuclear compartment, leading to microvessel leakage. Simultaneously, the rapid activation of Src by insulin followed by the activation of Rac1, a small GTPase involved in cytoskeletal reorganization, leads to the maintenance of endothelial barrier, short-circuiting the slower thrombin-induced Src-RhoA signaling that leads to endothelial permeability. Our results suggest that insulin treatment should be considered an approach to control vessel permeability and could be used in resuscitation treatments of extensive burns, sepsis, and other severe pathological conditions.

\section{Materials and Methods}

\section{Reagents}

Primary human dermal microvascular endothelial cells (HDMECs) were purchased from ScienCell Research Lab. (Carlsbad, CA, USA). A human microvascular endothelial cell line,
144

J Vasc Res 2017;54:143-155 DOI: $10.1159 / 000470844$
Liu/Chen/Wang/Martins-Green 
HMEC-1, was obtained as a gift from the Centers for Disease Control and Prevention (Atlanta, GA, USA). DMEM was purchased from Mediatech (Herndon, VA, USA), and FBS was from Atlanta Biologicals (Norcross, GA, USA). Transwell systems and Matrigel matrix were purchased from BD Biosciences (Franklin Lakes, NJ, USA), 3-kDa fluorescein isothiocyanate (FITC) dextran, rhodamine-phalloidin, and Alexa-Fluor 594 goat anti-rabbit antibody were from Invitrogen (Carlsbad, CA, USA). Src tyrosine kinase inhibitor pyrazolopyrimidine (PP2) was purchased from EMD4Biosciences (Gibbstown, NJ, USA), Rac1 inhibitor NSC 23766 from Cayman Chemical (Ann Arbor, MI, USA) and Rho kinase (ROCK) inhibitor Y-27632, broad tyrosine kinases inhibitor Genistein and insulin receptor (IR) neutralizing antibodies (IRAb) (29B4) were from Calbiochem (San Diego, CA, USA). Bovine thrombin was purchased from Fisher Bioreagents (Fair lawn, NJ, USA), recombinant human insulin for cell culture was from Sigma-Aldrich (St. Louis, MO, USA), and Humulin human insulin (rDNA origin) isophane suspension for in vivo animal experiment was from Eli Lilly and Company. Protein-G/Sepharose was from GE Healthcare, Life Sciences (Pittsburgh, PA, USA). The following antibodies were obtained from various suppliers: anti-phosphoY731 VE cadherin (Abcam, Cambridge, MA, USA), anti-VE cadherin, anti-phospho-Tyr416 Src, SHP2 and GAPDH (Cell signaling Technology, Danvers, MA, USA), anti-Rac1 (BD Biosciences, Franklin Lakes, NJ, USA), anti- $\beta$-Actin (Sigma-Aldrich, St. Louis, MO, USA), horseradish peroxidaseconjugated secondary antibodies (Thermo Scientific, Rockford, IL, USA), and FITC-conjugated goat anti-mouse immunoglobulin (Zymed Laboratories, South San Francisco, CA, USA). Rho Activation Assay Kit and Rac1 Activation Magnetic Beads Pull down Assay were purchased from EMD Millipore Corporation (Temecula, CA, USA).

\section{Cell Culture}

HDMEC were cultured with ECM containing growth supplements (ScienCell Research Lab.). HMEC-1 cells were cultured in $10 \%$ FBS DMEM. All cells were cultured in $5 \% \mathrm{CO}_{2}$ at $37^{\circ} \mathrm{C}$.

\section{In Vivo Permeability Assay}

C57/B6 mice were purchased from FuDan University (Shanghai, China), and housed at the Shanghai JiaoTong University, School of Medicine (SJTUSM). Experimental protocols were approved by the SJTUSM Animal Care and Use Committee. The mice were anesthetized with ketamine $(80 \mathrm{mg} / \mathrm{kg})$ and xylazine $(16$ $\mathrm{mg} / \mathrm{kg}$ ) (IP) at the start of the experiment. The in vivo permeability assay was conducted as described previously for VEGF [17]. C57/B6 mice (6-8 months old, $n=8$ ) were injected intravenously via the tail vein with $100 \mu \mathrm{L}$ of $2 \%$ Evans blue dye in sterile normal saline. After dye injection, contralateral regions of the mouse dorsum were injected subcutaneously with $25 \mu \mathrm{L}$ of vehicle (saline, NS), $0.03 \mathrm{U}$ insulin or $100 \mathrm{U}$ thrombin in $25 \mu \mathrm{L}$ of NS, or $0.03 \mathrm{U}$ insulin plus $100 \mathrm{U}$ thrombin in $25 \mu \mathrm{L}$ of NS. Thirty minutes after injection, mice were sacrificed by $\mathrm{CO}_{2}$ inhalation and perfused with sterile NS; a 7-mm punch biopsy was removed from the injected skin region. Skin punches were photographed. Evans blue was then extracted from these biopsies by incubation with $400 \mu \mathrm{L}$ of formamide at $56^{\circ} \mathrm{C}$ for $24 \mathrm{~h}$, and uptake was quantified using a spectrophotometer at $600 \mathrm{~nm}$ absorbance. Absorbance was normalized to tissue weight.

\section{In vitro Permeability Assays}

The procedure was conducted as previously described [18]. HDMECs were placed on Matrigel-coated transwell inserts with $3 \mu \mathrm{m}$ pore size. A total of $1 \times 10^{5}$ cells in $100 \mu \mathrm{L}$ of media were plated within transwell inserts. Three hours later, the media with nonadherent cells were removed, and $300 \mu \mathrm{L}$ of fresh media were added to the insert and $1 \mathrm{~mL}$ of media was added to the lower chamber. Twenty-four hours after plating, the same number of HDMECs were plated for the second time using the same protocol. Twentyfour hours after the second plating, an endothelial cell monolayer with optimal barrier function was formed. A quantity of $10 \mu \mathrm{g}$ of 3-kDa FITC-dextran was added to the lower chamber of the transwell system along with thrombin or thrombin plus insulin. Untreated cultures served as controls. For all permeability assays, 10 $\mu \mathrm{L}$ aliquots were removed at the indicated time points from the upper chamber, and fluorescence intensity was quantified using a fluorimeter (Victor 1420; PerkinElmer Life Sciences and Analytical Sciences, Boston, MA, USA), with excitation at $485 \mathrm{~nm}$ and emission at $535 \mathrm{~nm}$, to provide an indicator of relative endothelial permeability. Each treatment group was performed in triplicate.

\section{Immunolabeling}

Cells were cultured in $35 \mathrm{~mm}$ cell culture dishes, fixed in $4 \%$ paraformaldehyde for $20 \mathrm{~min}$, rinsed with PBS, incubated in PBS containing $0.1 \mathrm{M}$ glycine for $20 \mathrm{~min}$, and blocked with 3\% BSA, $0.01 \%$ Triton X-100 in PBS for 30 min. Primary antibodies in $1 \%$ $\mathrm{BSA} / \mathrm{PBS}$ were applied to the sample for $2 \mathrm{~h}$ at room temperature, washed, and incubated with 1:200 dilutions of FITC or Alexa Fluor 594-conjugated secondary antibodies for $1 \mathrm{~h}$ at room temperature. For actin staining, we used Rhodamine-phalloidin. After washing, the cells were mounted in Vectashield-containing DAPI (Vector Laboratories, Inc., Berlingame, CA, USA). Immunofluorescence was visualized and imaged using a Nikon microphotFXA microscope.

\section{Immunoblot Analysis}

Cells were treated as indicated, washed with ice-cold 1X PBS, and lysed on ice with lysis buffer containing $0.5 \%$ Triton X-100, 0.5\% Nonidet P-40, $10 \mathrm{mM}$ Tris, pH 7.5, $2.5 \mathrm{mM} \mathrm{KCl,} 150 \mathrm{mM}$ $\mathrm{NaCl}, 30 \mathrm{~mm}$ b-glycerophosphate, $50 \mathrm{~mm} \mathrm{NaF}, 1 \mathrm{mM} \mathrm{Na}_{3} \mathrm{VO}_{4}$, $0.1 \%$ SDS, and additional protease and phosphatase inhibitor cocktails. Protein concentrations were measured using the DC protein assay kit (Bio-Rad). Equal amounts of protein in the cell extracts were mixed with sample buffer, boiled, and analyzed using $10 \%$ acrylamide SDS-PAGE. Immunoblotting was performed with the appropriate primary antibodies and HRP-conjugated secondary antibodies, followed by incubation with West Dura extended duration substrate (Pierce Biotechnology). The Blot signals were captured either by X-ray film or using Image Lab software. Blots were then reprobed for total protein or housekeeping proteins to show equal loading.

\section{Rac1 and RhoA Activation Assay}

The procedure was conducted per manufacturer's instruction. In briefly, $10 \mu \mathrm{L}$ of the Racl Assay Reagent or $15 \mu \mathrm{L}$ of the Rho Assay Reagent were added into each cell extract. The cell extracts were then incubated for $45 \mathrm{~min}$ at $4^{\circ} \mathrm{C}$ with gentle agitation. The agarose beads were pellet, washed 3 times, and then resuspended and boiled for $5 \mathrm{~min}$. The supernatants and the agarose pellet were loaded for the SDS-PAGE and Western blot analysis. 
Trypsinization Experiments

To distinguish between cell surface and intracellular VE-cadherin, HMEC- 1 cells were rinsed with PBS and incubated in trypsin/EDTA at $37^{\circ} \mathrm{C}$ to proteolytically remove cell-surface VE-cadherin. Trypsin was subsequently neutralized using $10 \%$ FBS DMEM. Cells were then collected by centrifugation, and cell pellets were lysed with RIPA buffer. For controls, parallel cultures were harvested in RIPA buffer without trypsinization. Cell lyses were then analyzed by immunoblot analysis as described above.

\section{Detergent Fractionation}

The procedure was conducted as described previously [19] with some modifications. Briefly, cells were sequentially extracted with cytosol extraction buffer (10 mM PIPES, 5 mM EDTA, pH 6.8, $300 \mathrm{~mm}$ sucrose, $100 \mathrm{mM} \mathrm{NaCl}, 3 \mathrm{mM} \mathrm{MgCl}_{2}, 0.01 \%$ (w/v) digitonin), and membrane/organelle extraction buffer (10 mM PIPES, 3 mM EDTA, pH 7.4, $300 \mathrm{~mm}$ sucrose, $100 \mathrm{~mm} \mathrm{NaCl}, 3 \mathrm{mM} \mathrm{MgCl}_{2}$, $0.5 \%(\mathrm{v} / \mathrm{v})$ Triton X-100) for 10 and $20 \mathrm{~min}$. The material remaining on the plate was scraped in cytoskeletal/DNA extraction buffer (0.3\% SDS, $200 \mathrm{~mm}$ DTT, $28 \mathrm{~mm}$ Tris- $\mathrm{HCl}, 22 \mathrm{~mm}$ Tris base), collected, and boiled for $5 \mathrm{~min}$ to decrease the viscosity. All steps utilize approximately $20 \mu \mathrm{L} / \mathrm{cm}^{2}$ of ice-cold extraction buffer supplemented with freshly added protease inhibitor cocktail. Detergent extracts were then diluted with an equal volume of ice-cold distilled and de-ionized $\mathrm{H}_{2} \mathrm{O}$, and proteins precipitated with 4 volumes of ice-cold acetone by leaving the solution at $-20^{\circ} \mathrm{C}$ for 30 min. Protein pellets were redissolved in RIPA buffer. Protein concentrations were measured and equal amounts of protein were mixed with the appropriate volume of SDS-PAGE sample buffer then boiled in a water bath for 5 min with intermittent vortex mixing. Samples were then analyzed using 10\% acrylamide SDSPAGE.

\section{Immunoprecipitation}

After detergent fractionation, protein pellets were dissolved in $100 \mu \mathrm{L}$ RIPA buffer. Protein concentrations were measured and equal amounts of protein were used; RIPA buffer was added to make a total volume of $500 \mu \mathrm{L} /$ sample. A volume of $5 \mu \mathrm{L}$ of antiVE-cadherin antibody was added and then incubated at $4{ }^{\circ} \mathrm{C}$ for $2 \mathrm{~h}$. A quantity of $40 \mu \mathrm{L}$ of Protein-G/Sepharose was then added and rocked at $4{ }^{\circ} \mathrm{C}$ for $2 \mathrm{~h}$. Beads were then washed 3 times with $150 \mathrm{mM}$ RIPA buffer and $20 \mathrm{mM} \mathrm{NaCl}$ RIPA to equilibrate back to low salt. All supernatant were removed, $4 \times$ sample buffer was added, and samples were boiled for $5 \mathrm{~min}$; this was followed by 13,000 rpm centrifuge for $15 \mathrm{~min}$ to crush beads and release protein. Samples were then analyzed using 10\% acrylamide SDS-PAGE and perform immunoblotting.

\section{Statistical Analysis}

Data analysis was performed using GraphPad Instat software (GraphPad Software Inc.). $t$ tests were used to determine the significance of pair-wise differences between means, unpaired $t$ tests for comparison between 2 groups, and one-way ANOVA (Dunnett's post hoc test) were used to determine significance between means of several groups. Data satisfying the assumptions of ANOVA were verified before performing the tests. A $p$ value $<0.05$ was considered statistically significant, and a $p$ value $<0.01$ was considered highly statistically significant. Data are shown as mean \pm SD.

\section{Results}

Insulin Antagonizes Thrombin-Induced Blood Vessel Leakage and Permeability of Tight Endothelial Monolayers

The effects of insulin and/or thrombin on vessel permeability were examined by monitoring the extravasation of Evans blue dye from the circulation into the surrounding tissues after treatment with thrombin in the presence or absence of insulin. For the localized in vivo microvessel permeability assay in the skin, we chose a dose of insulin that does not significantly affect blood glucose levels based on our previous work [14]. For thrombin, the dose was determined in preliminary experiments that measured frank Evans blue extravasation. The dose we used was higher than the concentration of thrombin produced during inflammation in vivo [20]. After perfusion of Evans blue, thrombin or insulin or thrombin + insulin were injected under the skin in 4 different locations on the shaved back of a perfused mouse. Thrombin significantly increases permeability in vivo as shown by the Evans blue extravasation into the thrombin-treated tissue area. This effect was significantly inhibited by the presence of insulin (Fig. 1a). The results were confirmed by quantifying the Evans blue extracted from the different treated tissues (Fig. 1b).

To determine the molecular mechanisms involved in the inhibitory effects of insulin on thrombin-induced endothelial permeability, we used a system we have previously developed for in vitro permeability assays [17]. Primary HDMECs were treated with $1.5 \mathrm{U} / \mathrm{mL}$ thrombin or $1.5 \mathrm{U} / \mathrm{mL}$ thrombin plus $10^{-8} \mathrm{M}$ insulin. We have previously shown that at this low dose, insulin functions exclusively through the IR [14]. Thrombin treatment resulted in increased FITC-dextran movement from the lower chamber to the upper chamber, illustrating the increase in permeability, whereas this was prevented by simultaneous treatment with insulin (Fig. 1c), suggesting an antagonistic effect of insulin on thrombin-induced permeability of the "endothelium." Corresponding cell morphological changes and intercellular gap formation were visualized by F-actin staining (Fig. 1d). The numbers and sizes of gaps were significantly decreased when insulin was added with thrombin.

\section{Insulin Interferes with Thrombin-Induced}

VE-Cadherin-SHP2 Complex Translocation from the

Plasma Membrane to the Cytoskeleton

VE-cadherin plays a critical role in endothelial cell and microvessel permeability because it is involved in inter-
146
J Vasc Res 2017;54:143-155

DOI: $10.1159 / 000470844$
Liu/Chen/Wang/Martins-Green 
Fig. 1. Insulin antagonizes thrombin-induced blood vessel leakage and thrombininduced permeability in tight endothelial monolayers. a Mice were injected with Evans blue via tail vein, and then injected subcutaneously with either normal saline (NS) as vehicle, thrombin, insulin, or thrombin plus insulin. Thirty minutes after treatment, mice were euthanized and perfused with NS to clean the dye from the circulatory system, followed by the removal of treated regions of skin via punch biopsy. b Evans blue was extracted from the skin punches and quantified by spectrophotometry. Statistical analysis was performed as described in the Materials and Methods section; data are shown as mean \pm SD. $* p<0.05, n=8$. c, d Insulin antagonizes thrombin-induced blood vessel leakage and thrombin-induced permeability in tight endothelial monolayers: c Human dermal microvascular endothelial cells (HDMECs) were plated for permeability assays in Transwell culture systems and treated with $1.5 \mathrm{U} / \mathrm{mL}$ thrombin or $1.5 \mathrm{U} /$ $\mathrm{mL}$ thrombin plus $10^{-8} \mathrm{M} / \mathrm{mL}$ insulin as described in Materials and Methods. Each treatment was performed in triplicate $(\mathrm{n}=$ 3). Statistical analysis was performed as described in the Materials and Methods section; data are shown as mean $\pm \mathrm{SD}$. ${ }^{*} p<$ 0.05 (Ctrl vs. Thr, Thr vs. Thr + Ins). d HDMECs monolayers were either left untreated or treated as indicated on the Figure. The cells were immunostained with rhodamine-phalloidin to visualize paracellular gaps using fluorescence microscopy. Arrowheads point to endothelial gaps. Pictures are representative of 3 experiments. Scale bars $=20 \mu \mathrm{m}$.

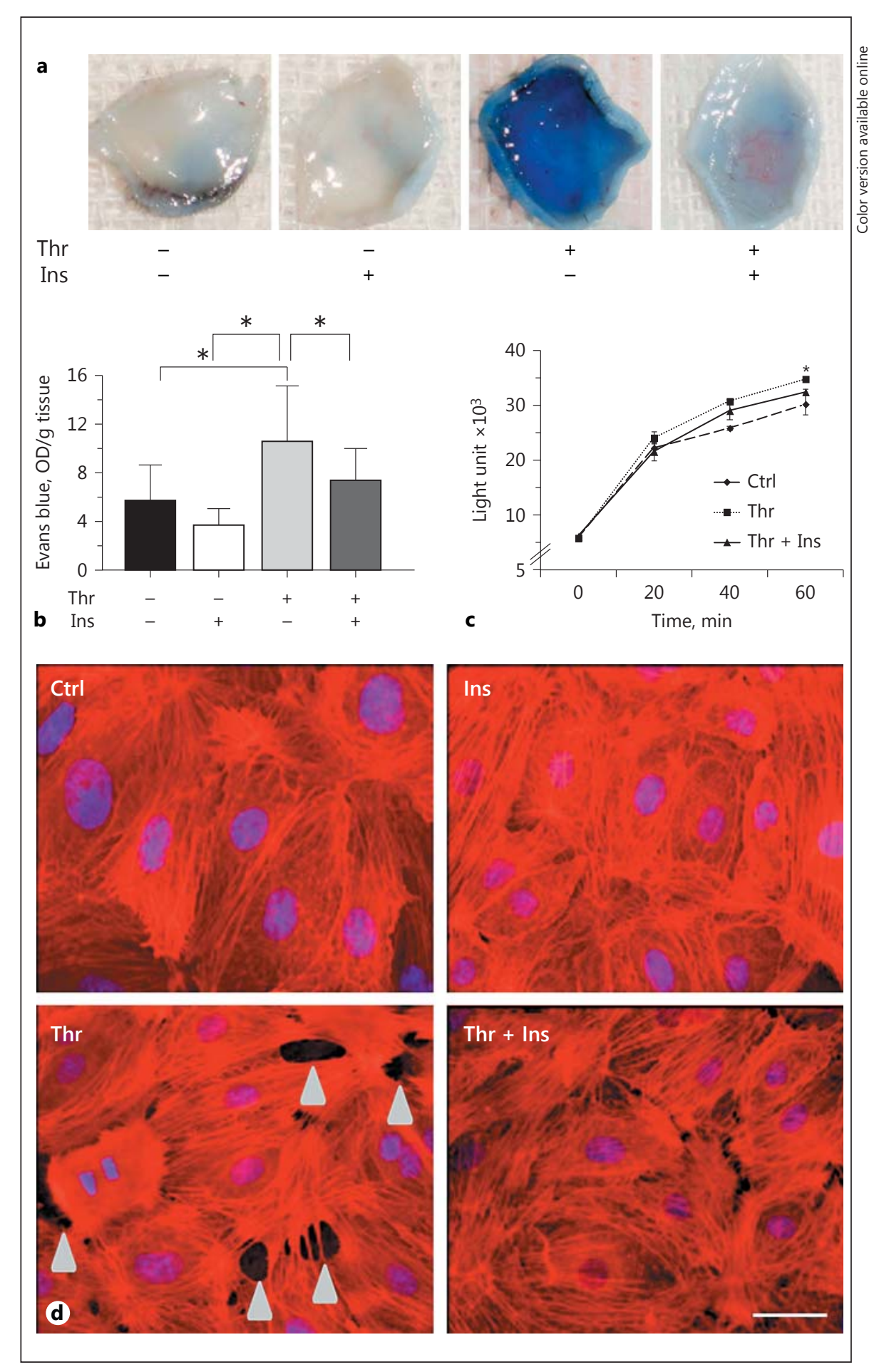

cellular adhesion junctions. It has been shown that thrombin induces activation/phosphorylation of VE-cadherin leading to permeability increase [21]. To detect whether insulin exerts an antagonistic effect by interfering with thrombin-induced phosphorylation of VE-cadherin, we examined VE-cadherin phosphorylation by thrombin alone and by thrombin in the presence of insulin. Increased phosphorylation of VE-cadherin at 3 and $5 \mathrm{~min}$ after thrombin treatment followed by decreased phosphorylation by 15 min suggests that thrombin induces 


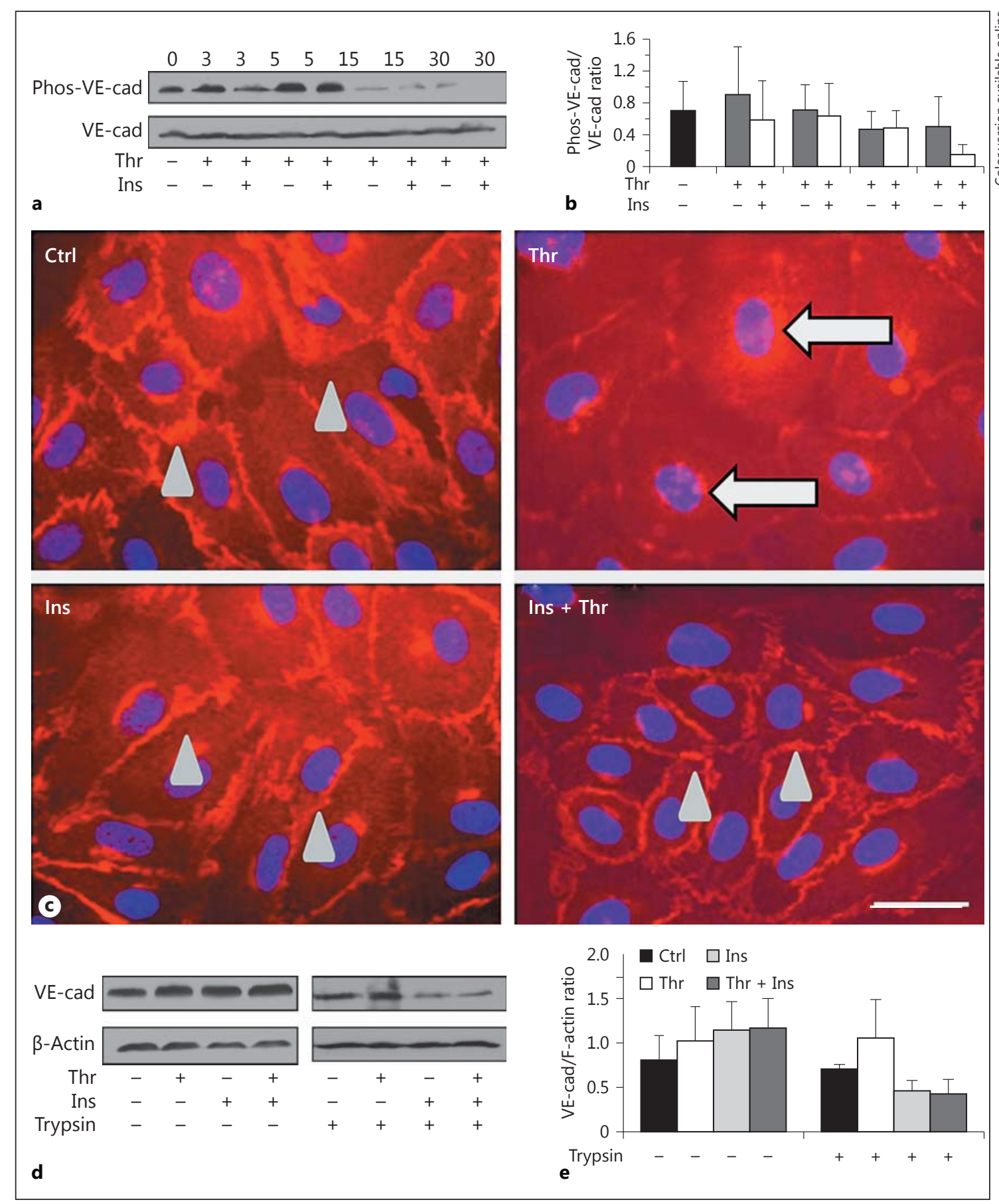

Fig. 2. Insulin interferes with thrombin-induced phosphorylation/ dephosphorylation of VE-cadherin. a HDMEC-1 cells were treated with thrombin or pretreated with $10^{-8} \mathrm{M}$ insulin for $5 \mathrm{~min}$ followed by thrombin treatment for the indicated time. b Densitometric quantification of phosphorylated VE-cadherin immunoblots using Image J software. Phos-VE-cadherin signals were normalized to VE-cadherin signal to account for variability in loading. Statistical analysis was performed as described in the Materials and Methods section; data are shown as mean \pm SD. $n=5$. c HDMEC were either left untreated or treated with $1.5 \mathrm{U} / \mathrm{mL}$ thrombin or $10^{-8} \mathrm{M}$ insulin or $1.5 \mathrm{U} / \mathrm{mL}$ thrombin plus $10^{-8} \mathrm{M}$ insulin for $5 \mathrm{~min}$ and then fixed and immunolabeling for VE-cadherin. Pictures are representative of 3 experiments. d Total VEcadherin and intracellular VE-cadherin was assessed by trypsinization experiments as described in Materials and Methods. e Densitometric quantification of VE-cadherin immunoblots using Image J software. VE-cadherin signals were normalized to Factin signal to account for variability in loading. Statistical analysis was performed as described in the Materials and Methods section; data are shown as mean \pm SD. ${ }^{*} p<0.05 . n=3$. Scale bars $=20 \mu \mathrm{m}$. 
rapid phosphorylation of VE-cadherin in the first few minutes after treatment but that this phosphorylation subsides quickly. We also observed a trend in decreasing of VE-cadherin phosphorylation when endothelial cells were pretreated with insulin, although that decrease was not statistically significant (Fig. 2a, b).

The activation and internalization of VE-cadherin are important for thrombin-induced vessel permeability [12]. Using immunolabeling to detect the cellular distribution of VE-cadherin we found that endothelial cell tight monolayers in the presence of insulin show VE-cadherin on the cell surface (Fig. 2c). Thrombin treatment increases the presence of VE-cadherin in the cytosol and perinuclear area with decreased presence in the plasma membrane (PM). However, when the cells were treated with thrombin in the presence of insulin pretreatment, there was no significant movement of $\mathrm{VE}$-cadherin to the cytosol (Fig. 2c). To confirm these findings quantitatively, we conducted trypsinization experiments to distinguish between cell surface and intracellular VE-cadherin. There is no significant difference in VE-cadherin levels between untreated cells and cells treated with thrombin, insulin, or thrombin combined with insulin (Fig. $2 \mathrm{~d}$ left, e). However, by incubating the cells with trypsin/EDTA, cell surface VE-cadherin should be proteolytically removed and only intracellular VE-cadherin should be detected. Thrombin results in a significant increase in VEcadherin because this protein was internalized and protected from trypsinization. However, when thrombin treatment was preceded by insulin pretreatment, there was a significant decrease in intracellular VE-cadherin because more of it stayed on the surface and was digested by the trypsin (Fig. $2 \mathrm{~d}$ right, e). These results suggest that insulin reverses the effects of thrombin on VE-cadherin distribution and that thrombin-induced VE-cadherin translocation to the cytosol is inhibited by insulin. Detergent fractionation assays that were performed as previously described [19] confirmed these findings. Thrombin and/or insulin pretreated endothelial cells were fractionated into cytosolic, cell membrane and cytoskeletal/nuclear compartments and the extracts analyzed by immunoblotting for VE-cadherin. Thrombin treatment increased the levels of VE-cadherin present in the cytosol and in the cytoskeletal/nuclear fraction (Fig. 3a). However, when the cells are pretreated with insulin before exposure to thrombin, less VE-cadherin was seen in the cytosol and cytoskeleton/nuclear fraction but more was present on the PM (Fig. 3a, b).

Because thrombin is known to cause the activation and dissociation of SHP2 from VE-cadherin/SHP2/ $\beta$-catenin

Insulin Increases Microvessel Stability complex, we then used cells fractionation to immunoprecipite VE-cadherin and detected the levels of VE-cadherin associated SHP2. We found significant increase in VE-cadherin and VE-cadherin associated SHP2 in cytoskeletal/nuclear fraction of thrombin-treated cells but not in thrombin-treated cells pretreated with insulin. Correspondingly, much less VE-cadherin was visibly detected in the membrane fraction of thrombin treatment cells but not thrombin plus insulin-treated cells (Fig. 3c).

Taken together, these results indicate that the antagonistic effect of insulin on thrombin-induced endothelial cell permeability may be due to insulin interfering with thrombin-induced activation/internalization of VE-cadherin. Contrary to what has been previously reported we did not find obvious disassociation of SHP2 from VE-cadherin on the cell membrane upon thrombin treatment. It appears that thrombin induces translocation of SHP2-VEcadherin complex from the membrane to the cytoskeleton, and insulin interfered with this translocation process and keeps more VE-cadherin and SHP2 on the cell membrane.

Small GTPase Rac1 is Involved in the Antagonistic Effect of Insulin on Thrombin-Induced Permeability and Src Phosphorylation is Important in this Process

Small GTPase RhoA and Racl regulate cell contraction by regulating Myosin Light Chain (MLC) phosphorylation and actin stress fiber formation. RhoA has been reported to be involved in thrombin-induced increase in vessel permeability [22] through its association with ROCK [23]. We observed no gap formation when the cells were treated with thrombin plus ROCK inhibitor confirming these previous results. The absence of gap formation was also observed when the cells were pretreated with insulin followed by thrombin in the presence of the ROCK inhibitor (Fig. 4a).

We have previously shown that insulin stimulates keratinocyte and endothelial cell migration through Rac1 $[14,24]$. In this study, we show that Rac1 inhibitor, NSC 23766, does not interfere with thrombin-induced gap formation, including when insulin is present (Fig. 4b), suggesting that the antagonistic effect of insulin on thrombin-induced vessel permeability is mediated by Rac1. These results were further supported by Rac1 immunolabeling (Fig. 4c). Homogeneous intracellular distribution of Racl was found in control and thrombin-treated cells, whereas insulin treatment caused significant increase of Rac1 localization in the peri-nuclear area. Similar Rac1 peri-nuclear distribution was observed in thrombin plus insulin-treated cells (Fig. 4c). We further conducted RhoA and Racl activation assays, and found that the

J Vasc Res 2017;54:143-155 149 
Fig. 3. Insulin interferes with thrombin-induced VE-cadherin-SHP2 complex translocation from the plasma membrane to the cytoskeleton. a Distribution of VE-cadherin in cytosol, cell membrane, and in cytoskeleton/nuclear fractions was monitored using detergent fractionation. b Densitometric quantification of VE-cadherin immunoblots using Image J software. VEcadherin signals were normalized to F-actin signal to account for variability in loading. Statistical analysis was performed as described in the Materials and Methods section; data are shown as mean \pm SD. ${ }^{*} p<$ 0.05 (Ctrl vs. Thr). $n=3$. c HDMEC- 1 cells left either untreated or treated with $1.5 \mathrm{U}$ thrombin for $5 \mathrm{~min}$ or pretreated with $10^{-8}$ $\mathrm{M}$ insulin for $5 \mathrm{~min}$ followed with $1.5 \mathrm{U}$ thrombin treatment for $5 \mathrm{~min}$ were fractionated, and different cellular portion were then immunoprecipitated with VEcadherin antibody, and followed with immunoblotting and probed with anti-VEcadherin and anti-SHP2 antibodies. The results are representative of at least $3 \mathrm{ex}$ periments.

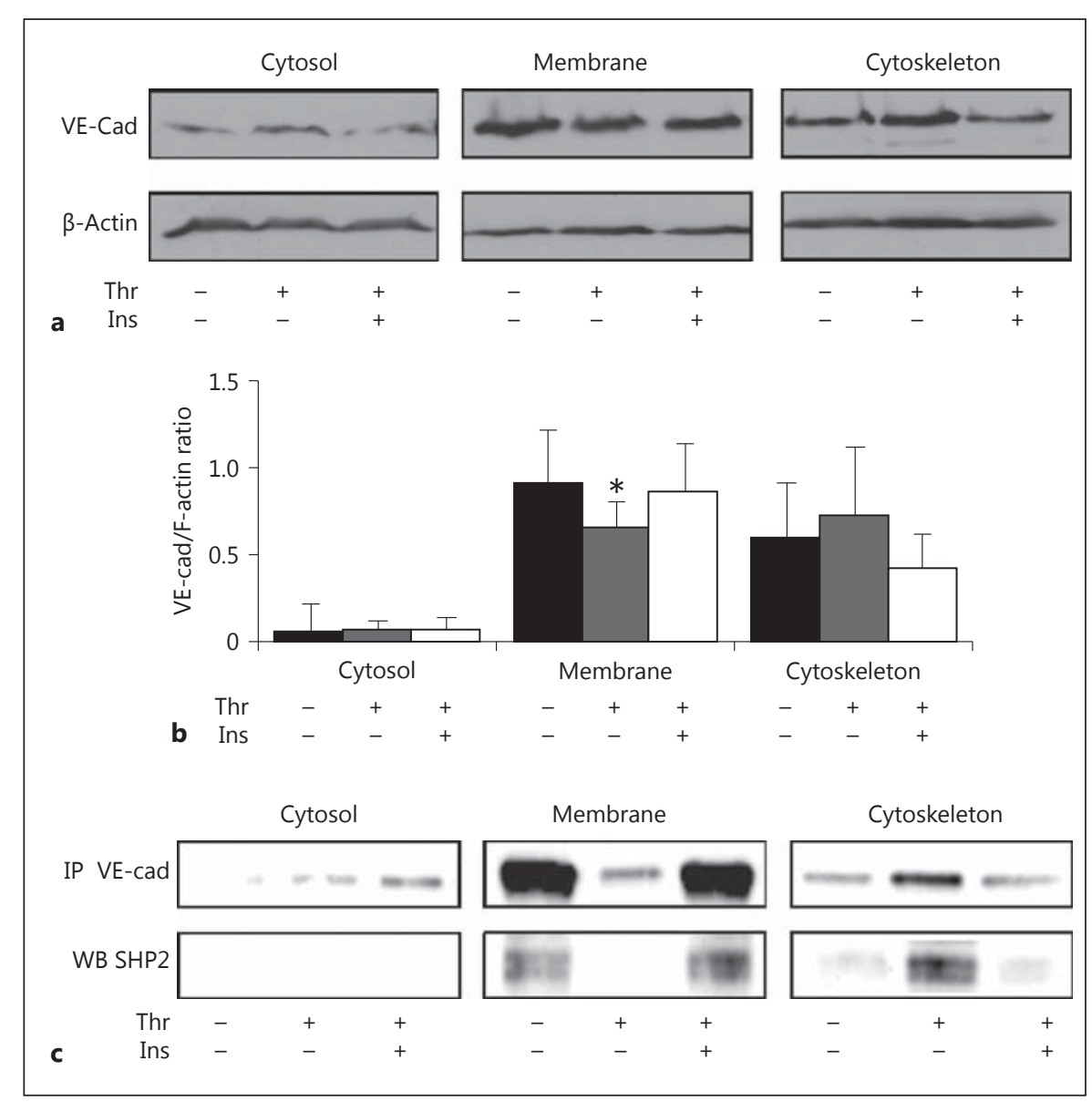

thrombin-induced RhoA activation was decreased by insulin (Fig. 4d). On the other hand, thrombin showed no effect on Racl activation, whereas insulin treatment alone or in combination with thrombin caused an increase in the Rac1 activation (Fig. 4e, f).

It has been reported that Src is important in thrombininduced vessel permeability [22]. We examined Src phosphorylation by immunoblot analysis and found that both thrombin (Fig. 5a) and insulin (Fig. 5b) treatment resulted in elevated phosphorylation of Src. However, insulin activates Src as early as 1 min after treatment, whereas significant Src activation is not seen until $5 \mathrm{~min}$ after stimulation of the cells by thrombin. We then examined Racl distribution and activation while Src activity was inhibited. The inhibition of Src by PP2 interfered with insulin-induced Rac1 activation (Fig. 5c, d) and with perinuclear Rac1 distribution. Decreased Rac1 distribution around the nuclear area is reduced in insulin or thrombin plus insulin-treated cells (Fig. 5e), suggesting that Src is involved in insulin-induced Rac1 activation.

\section{Discussion}

Our previous work showed that insulin stimulates endothelial cell migration through the PI3K-Akt-Rac1 signal pathway [14] and it stimulates angiogenesis and increases the number of aSMA-expressing cells associated with the microvessels, indicating an increase in vessel stability. Subsequent to the publishing of our work, others showed that the small GTPase Racl is important in controlling microvessel permeability [15], and confirmed that insulin stabilizes microvascular endothelial barrier function via phosphatidylinositol 3-kinase/Akt-mediated Rac1 [16]. Based on these findings, we tested the possibility that insulin inhibits increased microvascular permeability induced by inflammatory agents. We show above that local injection of insulin under the skin significantly inhibits thrombin-induced microvessel permeability, suggesting that insulin antagonizes thrombin-induced increase in permeability of microvessels. Specifically, insulin prevents thrombin-induced VE-cadherin 


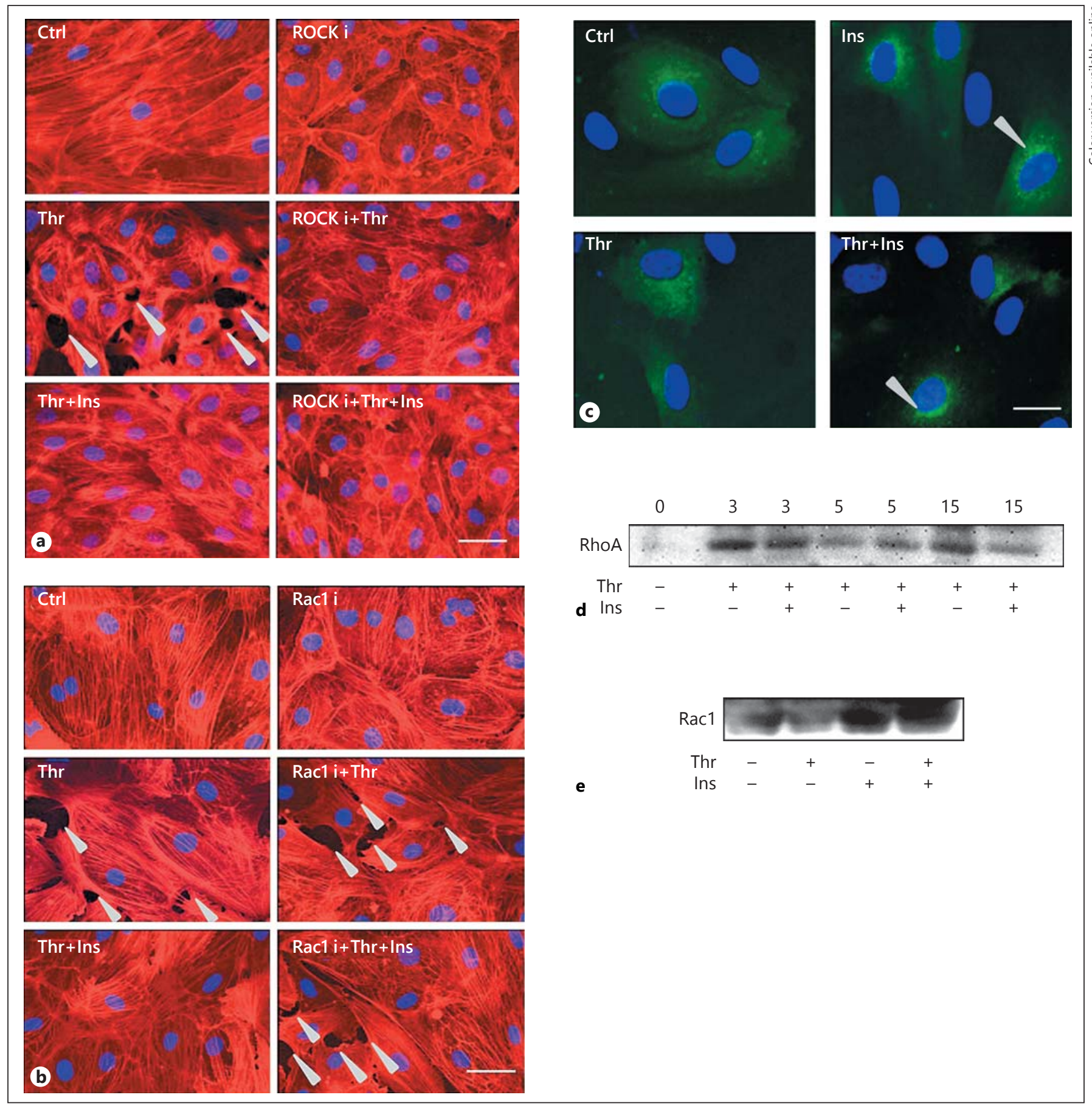

Fig. 4. Small GTPase Rac1 is involved in the antagonistic effect of insulin on thrombin-induced permeability. a, b HDMEC were either left untreated or pretreated with $1 \mu \mathrm{M}$ ROCK inhibitor Y-27632 (a) or $50 \mu \mathrm{M}$ Rac1 inhibitor NSC23766 (b) for $30 \mathrm{~min}$ followed by thrombin or insulin or thrombin plus insulin treatment for $15 \mathrm{~min}$. Cells were then fixed and stained with rhodaminephalloidin. c HDMECs were either left untreated, or treated with thrombin or insulin or thrombin plus insulin for $5 \mathrm{~min}$. Racl distribution was visualized by immunolabeling with an antibody to Rac1. a-c Representative of 3 experiments. Scale bars $=20 \mu \mathrm{m}$.

Insulin Increases Microvessel Stability

J Vasc Res 2017;54:143-155

d HDMECs were either left untreated, or treated with thrombin or thrombin plus insulin for the indicated time. RhoA activation was measured using the RhoA Activation Assay Kit. e, f HDMECs were either left untreated, or treated with thrombin or insulin or thrombin plus insulin for 5 min. e Racl activation was measured using the Rac1 Activation Assay Kit. f Densitometric quantification of Racl immunoblots using Image J software. Statistical analysis was performed as described in the Materials and Methods section; data are shown as mean \pm SD. $n=3$. 


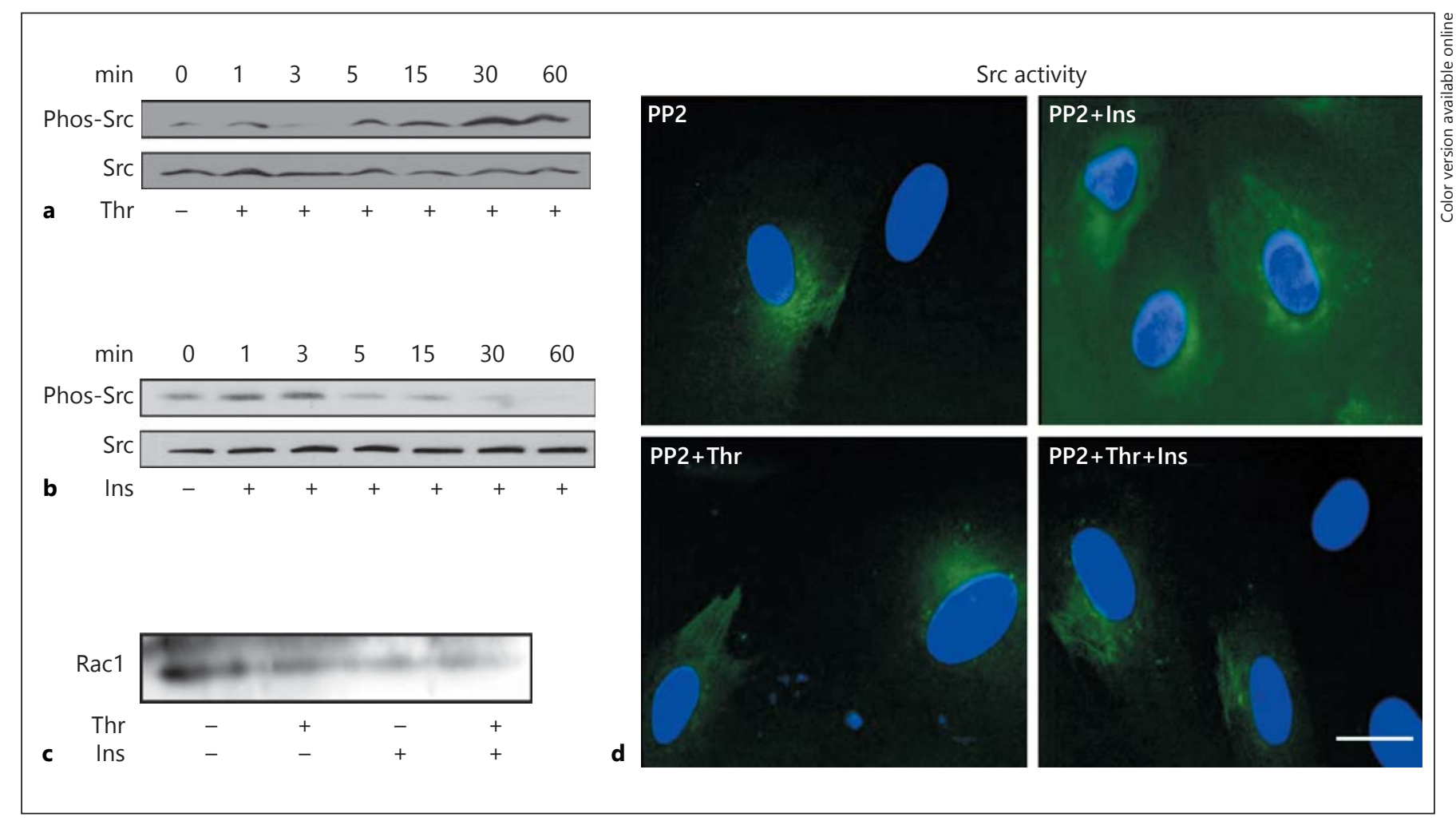

Fig. 5. Src is involved in the antagonistic effect of insulin on thrombin-induced permeability. a, b HDMEC-1 cells were either left untreated or treated as indicated, followed by immunoblot analysis of cell lysates using an antibody for phosphorylated Src. The blots were reprobed with anti-Src antibody to ensure equal loading. a Thrombin-induced Src phosphorylation. b Insulin-induced Src phosphorylation. c, d HDMEC-1 cells were pretreated with $10 \mu \mathrm{M}$ Src inhibitor PP2 for $1 \mathrm{~h}$, and then treated with thrombin or insulin or thrombin plus insulin for $5 \mathrm{~min}$. c Racl activation was mea- sured using Rac1 Activation Assay Kit. d Densitometric quantification of Rac1 immunoblots using Image J software. Statistical analysis was performed as described in the Materials and Methods section; data are shown as mean \pm SD. $* p<0.05 . n=3$. E HDMECs were pretreated with $10 \mu \mathrm{M}$ Src inhibitor PP2 for $1 \mathrm{~h}$, and then treated with thrombin or insulin or thrombin plus insulin for 5 min, Racl distribution was visualized by immunolabeling with an antibody to Rac1. a-c Representative of 3 experiments. Scale bars $=20 \mu \mathrm{m}$. translocation from the cell membrane to the cytosol. In the presence of insulin treatment, SHP2 (Src-homology 2 domain-containing phosphatase 2), an enzyme that is part of the adherent junction complex, remains associated with VE-cadherin in the PM, resulting in inhibition of gap formation and increase in permeability (Fig. 6).

VE-cadherin is a transmembrane protein that brings neighboring epithelial cells together to form cell-cell adherens junctions that contribute to the semi-permeable barrier function of endothelium. SHP2 is a tyrosine phosphatase that plays major roles in response to various factors that stimulate increase in microvessel permeability [25]. It has been shown that dissociation of SHP2 from the VE-cadherin complexes correlates with a dramatic increase in human umbilical vein endothelium permeability changes [26]. We show here that SHP2-VE-cadherin complex responds to thrombin by internalization (moving into the cytoskeleton/nuclear compartment) but not dissociation. We show that when endothelial cells are treated with thrombin in the presence of insulin pretreatment, SHP2 remains in the membrane, suggesting that insulin inhibits thrombin-induced increase in microvessel permeability by keeping SHP2 associated with the VEcadherin complex in cell membrane. SHP2 also plays an important role in the recovery of disrupted endothelial cell-cell junctions by dephosphorylating VE-cadherin and promoting the stability of VE-cadherin at the PM [27]. This may also occur in insulin-induced vessel permeability.

It is well known that GPCRs activate the Src family of kinases and that Src activity plays a role in controlling GPCR trafficking and cytoskeletal rearrangement [28]. 


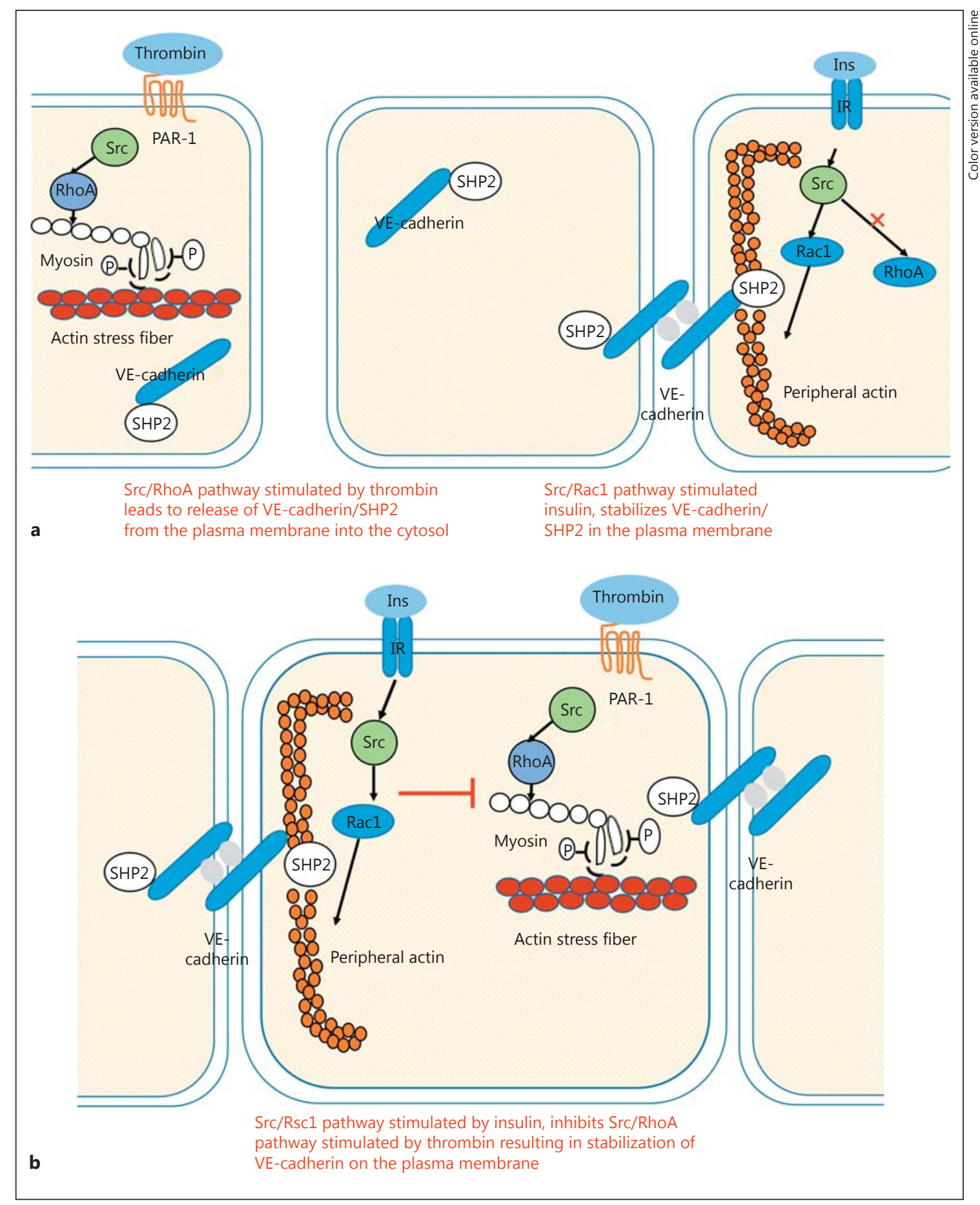

Fig. 6. Schematic representation of the mechanisms by which insulin inhibits thrombin-induced permeability. a Left cell. Thrombin stimulates the Src/RhoA signaling pathway leading to activation of the VE-cadherin-SHP2 complex and its release into the cytosol, leading to gap formation. a Middle cell. On the left shows VE-cadherin-SHP2 is released into the cytosol after thrombin activation and on the right side VE-cadherin-SHP2 remains on the membrane due to insulin signaling. a Right cell. Insulin treatment causes SHP2 to remain associated with VE-cadherin in the plasma membrane through the quick activation of Src/Racl signaling resulting in inhibition of gap formation. $\mathbf{b}$ Insulin inhibits thrombin signaling by rapidly activating the $\mathrm{Src} / \mathrm{Rac} 1$ signaling pathway and in that manner preventing the Src/RhoA thrombin-activated pathway, thereby preventing thrombin-induced activation of VE-cadherin-SHP2 to translocate from the cell membrane to the cytosol and consequently inhibiting endothelial cell permeability. PAR, proteolytic activated receptor; IR, insulin receptor; PM, plasma membrane. 
Src is involved in the thrombin-activated small GTPase RhoA. RhoA activation causes cell contraction by regulating MLC phosphorylation and actin stress fiber formation [23] Insulin-activated Src induces the activation of the small GTPase Racl and prevents cell contraction. Both insulin and thrombin activate Src, but they do so in different time frames. Insulin induces a rapid and brief activation of Src, whereas a delayed but lasting activation of Src was found after thrombin treatment. The difference in the time of activation between insulin and thrombin puts insulin in the driver's seat that targets Src before thrombin does. Small GTPases are a group of molecules involved in the regulation of the cytoskeleton. RhoA and Rac1 are both members of this family of molecules. Although RhoA activation is important in thrombin-induced vessel permeability, Racl activation is primarily related to the stabilization of the endothelium through the enhancement of the peripheral actin cytoskeleton, thus strengthening adherent junctions [14]. It has been shown in hemorrhagic shock that the balance in the activity of RhoA and Racl is important [28]. RhoA regulates the vascular permeability primarily through the activation of ROCK and inhibition of Rac1, whereas Rac1 activation significantly decreases the activity of RhoA [29] suggesting an intrinsic regulation between Racl and RhoA. Our data suggest that insulin-induced activation of Rac1 impairs the activation of RhoA, thus inhibiting thrombin-induced vessel permeability. Our findings suggest that the effects of insulin on Src and Racl activation are dominant over those of thrombin resulting in a vessel/ endothelial stabilization effect. Therefore, insulin, which has been safely used in the clinic for more than 100 years, is a promising candidate to control permeability induced by pathological conditions that involve thrombin release.

Although Src is important in the signaling that leads to the loss of barrier function of endothelium, the extensive biological effects in which Src is involved make its inhibition an unfeasible treatment. We show here that the Srcmediated antagonistic effect of insulin on thrombin-induced vessel permeability achieves the Src effects on barrier function without the side effects that would occur with Src inhibition.

In conclusion, under inflammatory conditions, insulin interferes with thrombin-induced VE-cadherin signaling by decreasing the ability of thrombin to induce the disassociation of the SHP2-VE-cadherin complex from the $\mathrm{PM}$, and translocation to the cytoskeleton/nuclear compartment, leading to microvessel leakage. Simultaneously, the rapid activation of Src by insulin followed by the activation of Rac1, a small GTPase involved in cytoskeletal reorganization, leads to the maintenance of the endothelial cell barrier, short-circuiting the slower thrombininduced Src-RhoA signaling that would have led to endothelial permeability. Our findings provide support for the use of insulin treatment in severe pathological conditions that involve blood barrier dysfunction.

\section{Acknowledgments}

We thank the Genomic Center for making the luminometer available for these studies. We also thank Melissa Petreaca, Neema Adhami and other colleagues in the Martins-Green laboratory for helpful discussions and help in submitting this manuscript.

This work was supported by the National Natural Science Fund of China (81170761 and 81270909 to Y.L.) and the National Institutes of Health to M.M.-G. (R21 AI078208).

\section{Disclosure Statement}

The authors have no conflicts of interest to disclose.

\section{References}

1 Petäjä J: Inflammation and coagulation An overview. Thromb Res 2011;127(suppl 2):S34-S37.

2 Gando S, Sawamura A, Hayakawa M: Trauma, shock, and disseminated intravascular coagulation: lessons from the classical literature. Ann Surg 2011;254:10-19.

3 Lippi G, Ippolito L, Cervellin G: Disseminated intravascular coagulation in burn injury. Semin Thromb Hemost 2010;36:429-436.
4 Tripodi A, Branchi A, Chantarangkul V, Clerici M, Merati G, Artoni A, Mannucci PM: Hypercoagulability in patients with type 2 diabetes mellitus detected by a thrombin generation assay. J Thromb Thrombolysis 2011;31: 165-172.

5 Huang Y, Yang Z: Measures for preventing early postburn damage improve survival rate of burn patients. Burns 2004;30:808-812.
6 Chai JK, Sheng ZY: [The present strategy and ponderation on prevention and treatment of burn sepsis and multiple organ dysfunction syndrome (MODS)]. Zhonghua Shao Shang Za Zhi 2008;24:378-380.

7 Martin GS, Mannino DM, Eaton S, Moss M: The epidemiology of sepsis in the United States from 1979 through 2000. N Engl J Med 2003;348:1546-1554.

8 Brun-Buisson C: The epidemiology of the systemic inflammatory response. Intensive Care Med 2000;26:S64-S74. 
9 Alberelli MA, De Candia E: Functional role of protease activated receptors in vascular biology. Vascul Pharmacol 2014;62:72-81.

10 Sukriti S, Tauseef M, Yazbeck P, Mehta D: Mechanisms regulating endothelial permeability. Pulm Circ 2014;4:535-551.

11 Coughlin SR: Thrombin signalling and protease-activated receptors. Nature 2000;407: 258-264.

12 Dejana E, Orsenigo F, Lampugnani MG: The role of adherens junctions and VE-cadherin in the control of vascular permeability. J Cell Sci 2008; 121:2115-2122.

13 Dellinger RP, Levy MM, Carlet JM, Bion J, Parker MM, Jaeschke R, Reinhart K, Angus DC, Brun-Buisson C, Beale R, et al: Surviving sepsis campaign: international guidelines for management of severe sepsis and septic shock: 2008. Crit Care Med 2008;36:296-327.

14 Liu Y, Petreaca M, Martins-Green M: Cell and molecular mechanisms of insulin-induced angiogenesis. J Cell Mol Med 2009; 13:44924504.

15 Spindler V, Schlegel N, Waschke J: Role of GTPases in control of microvascular permeability. Cardiovasc Res 2010;87:243-253.

16 Gündüz D, Thom J, Hussain I, Lopez D, Härtel FV, Erdogan A, Grebe M, Sedding D, Piper HM, Tillmanns $\mathrm{H}$, et al: Insulin stabilizes microvascular endothelial barrier function via phosphatidylinositol 3-kinase/Akt-mediated Racl activation. Arterioscler Thromb Vasc Biol 2010;30:1237-1245.
17 Petreaca ML, Yao M, Liu Y, Defea K, MartinsGreen M: Transactivation of vascular endothelial growth factor receptor- 2 by interleukin-8 (IL-8/CXCL8) is required for IL-8/ CXCL8-induced endothelial permeability. Mol Biol Cell 2007;18:5014-5023.

18 Martins-Green M, Petreaca M, Yao M: An assay system for in vitro detection of permeability in human "endothelium." Methods Enzymol 2008;443:137-153.

19 Chiang ET, Lim MJ, Patton WF, Shepro D: NFkappaB translocation in human microvessel endothelial cells using a four-compartment subcellular protein redistribution assay. J Biochem Biophys Methods 2000;46:53-68.

20 Mavrommatis AC, Theodoridis T, Economou M, Kotanidou A, El Ali M, Christopoulou-Kokkinou V, Zakynthinos SG: Activation of the fibrinolytic system and utilization of the coagulation inhibitors in sepsis: comparison with severe sepsis and septic shock. Intensive Care Med 2001;27:1853-1859.

21 Dejana E, Orsenigo F, Lampugnani MG: The role of adherens junctions and VE-cadherin in the control of vascular permeability. J Cell Sci 2008;121(pt 13):2115-2122.

22 Vouret-Craviari V, Bourcier C, Boulter E, van Obberghen-Schilling E: Distinct signals via Rho GTPases and Src drive shape changes by thrombin and sphingosine-1-phosphate in endothelial cells. J Cell Sci 2002;115:24752484 .
23 Van Nieuw Amerongen GP, Beckers CM, Achekar ID, Zeeman S, Musters RJ, van Hinsbergh VW: Involvement of Rho kinase in endothelial barrier maintenance. Arterioscler Thromb Vasc Biol 2007;27:2332-2339.

24 Liu Y, Petreaca M, Yao M, Martins-Green M: Cell and molecular mechanisms of keratinocyte function stimulated by insulin during wound healing. BMC Cell Bio 2009; 10:1.

25 Ukropec JA, Hollinger MK, Salva SM, Woolkalis MJ: SHP2 association with VE-cadherin complexes in human endothelial cells is regulated by thrombin. J Biol Chem 2000;275: 5983-5986.

26 Grinnell KL, Casserly B, Harrington EO: Role of protein tyrosine phosphatase SHP2 in barrier function of pulmonary endothelium. Am J Physiol Lung Cell Mol Physiol 2010; 298:L361-L370.

27 Timmerman I, Hoogenboezem M, Bennett AM, Geerts D, Hordijk PL, van Buul JD: The tyrosine phosphatase SHP2 regulates recovery of endothelial adherens junctions through control of $\beta$-catenin phosphorylation. Mol Biol Cell 2012;23:4212-4225.

28 Luttrell DK, Luttrell LM: Not so strange bedfellows: G-protein-coupled receptors and Src family kinases. Oncogene 2004;23:79697978.

29 Li T, Fang Y, Yang G, Xu J, Zhu Y, Liu L: Effects of the balance in activity of RhoA and Rac1 on the shock-induced biphasic change of vascular reactivity in rats. Ann Surg 2011; 253:185-193.

Insulin Increases Microvessel Stability 